\title{
Rectal inflammatory fibroid polyp in a Nigerian: case report \& brief review of literature.
}

\author{
Oluyemi Aderemi ${ }^{1}$, Awolola Nicholas ${ }^{2}$
}

1. ReMay Consultancy \& Medical Services, No 5 John Olugbo Street

2. College of Medicine, University of Lagos

\begin{abstract}
Background: Inflammatory fibroid polyps are very unusual gastrointestinal tumours. Furthermore, their occurrence in the rectum is a rarity indeed.

Objective: To document this very rare pathologic entity in a patient from our region of Africa and highlight some pertinent clinical and pathologic aspects of the disease.

Case report: We present a 59 year old Nigerian who had neither significant previous medical history nor current complaint and had a routine colonoscopy done. The finding in the rectum was of a small polypoidal mass which was excised whole and whose histopathologic findings were consistent with a diagnosis of inflammatory fibroid polyp.

Conclusion: A unique case of rectal inflammatory fibroid polyp in a Nigerian is hereby presented. This, to the best of our knowledge, is the first such documented case from our region of the world. Hence, the entity should be considered in the differential diagnosis of colonic polyps from the continent.

Keywords: Inflammatory fibroid polyps, colonoscopy, immunohistochemistry, Nigeria.

Doi: http://dx.doi.org/10.4314/ahs.v16i3.31

Cite as: Aderemi $O$, Nicholas A. Rectal inflammatory fibroid polyp in a Nigerian: case report \& brief review of literature. Afri Health Sci 2016;16(3): 873-876. DOI: http:// dx.doi.org/10.4314/abs.v16i3.31
\end{abstract}

\section{Introduction}

Inflammatory fibroid polyps (IFP) are rare submucosal growths in the gastrointestinal tract. They have no known malignant or metastatic potential and as such, currently, are thought to be entirely benign ${ }^{1}$.

These lesions are increasingly being picked up in the absence of symptoms due to the increasing use of endoscopy particularly in the colon ${ }^{2}$. Their presence can, however, lead to clinical symptoms and signs that might require endoscopic or surgical intervention ${ }^{2,3}$.

They are most commonly located in the stomach and small intestine when they occur along the gastrointestinal tract (GIT). Though large bowel lesions have been de-

\section{Corresponding author:}

Oluyemi Aderemi,

ReMay Consultancy \& Medical Services,

No 5 John Olugbo Street,

Email: mayremson@gmail.com scribed for virtually every region, the ano-rectum appears to be the only rarely involved ${ }^{4,5}$.

The case presented here documents, for perhaps the first time, one such rectal IFP in a Nigerian. The article will also discuss a few pertinent clinical and diagnostic aspects of the condition.

\section{Case report}

A 59 year old man presented for screening colonoscopy at a Lagos, Nigeria based endoscopy suite. The man was asymptomatic and had just been referred for the procedure by his general practitioner as a routine test.

A small, $3 \mathrm{~mm}$ in diameter polypoidal lesion was seen in the rectum (Figure 1). 


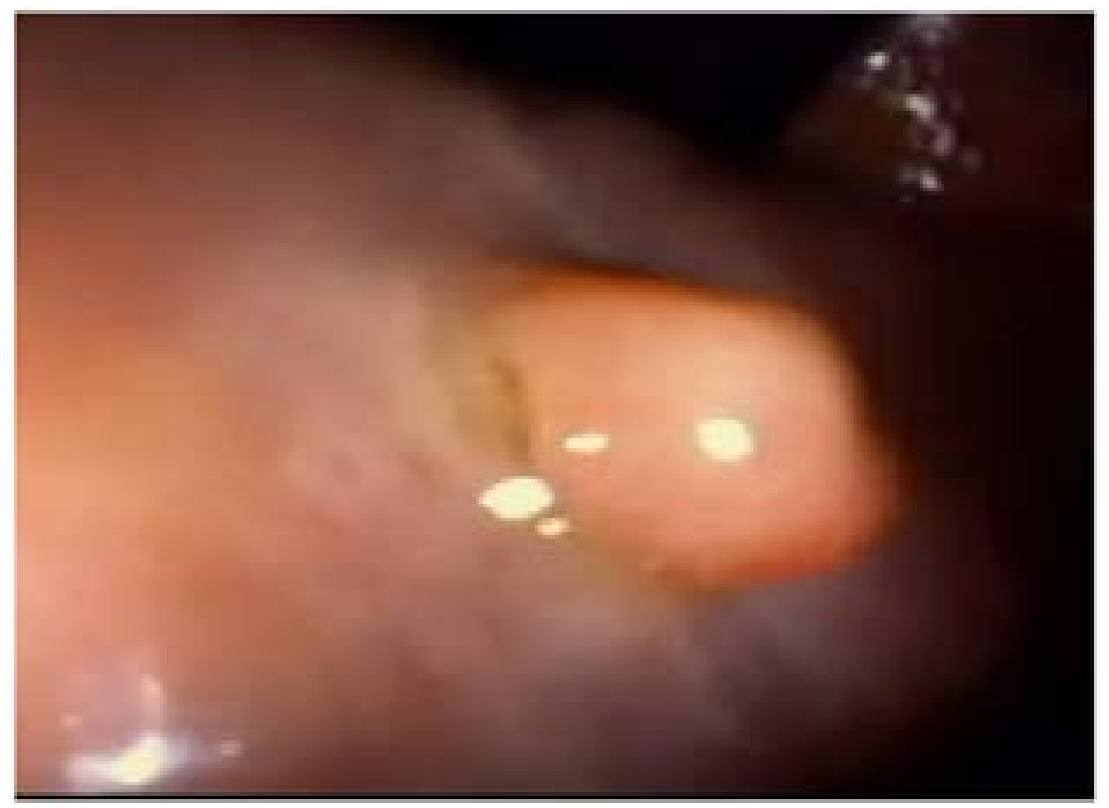

Figure 1: Endoscopic image of the rectal polyp.

The pinkish growth had a smooth surface with visible dilated blood vessels. The mass was removed whole with a cold snare biopsy forceps. Histopathologic report of the tissue showed submucosal populations of spindle cell lesion in fibrovascular stroma with various inflammatory infiltrates especially eosinophils. It was sparsely cellular with myxoid stroma and had zones of connective tissue around muscular walled blood vessels (Figure 2). Thus a diagnosis of rectal IFP made.

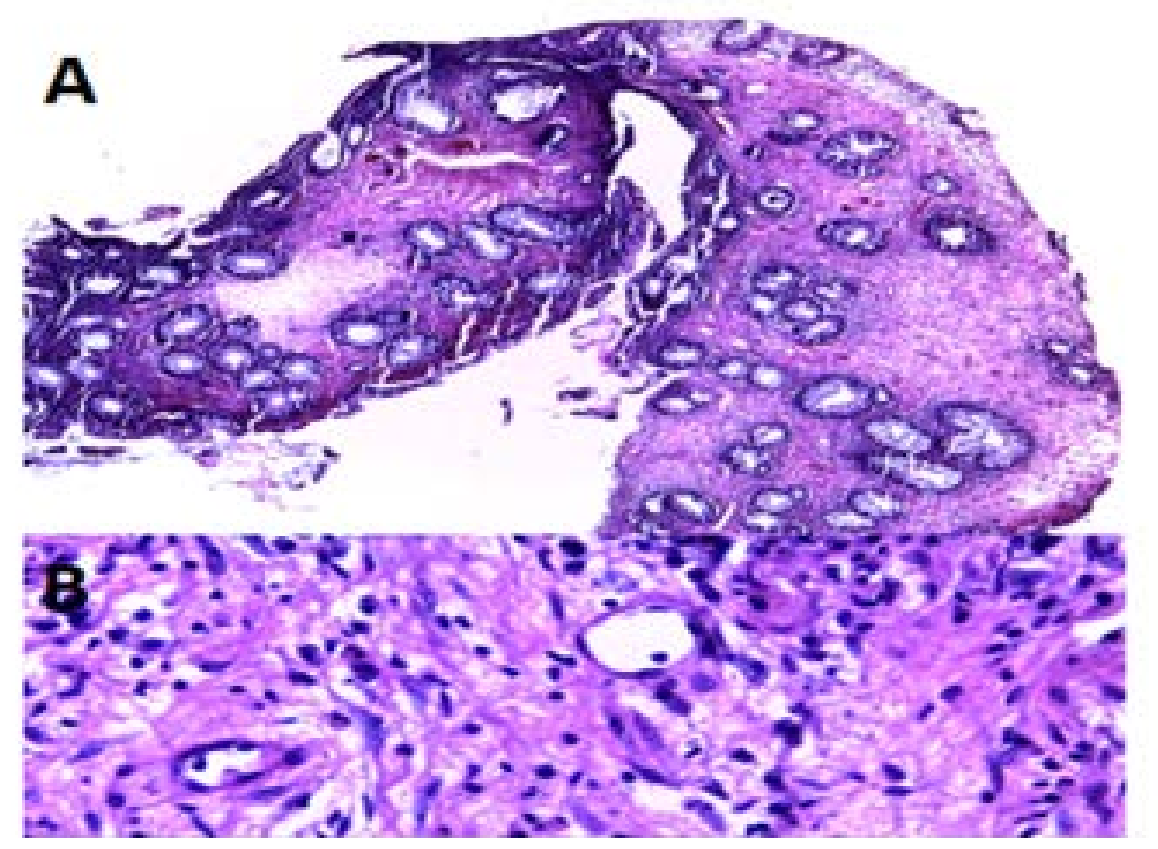

Figure 2: (A) Low power photomicrograph of inflammatory fibroid polyp (Hematoxylin and Eosin stain; original magnification $\times 40$ ). (B) Higher power photomicrograph of same section as $A$, showing concentric fibrosis around vessels(up arrow)and scattered eosinophils (right arrow) a midst other inflammatory cells(Hematoxylin and Eosin stain; original magnification $\times 400$ ). 


\section{Discussion}

Helwig and Ranier were the first to coin the term "inflammatory fibroid polyp" (IFP) in $1953^{6}$ to describe the entity previously referred to as "Vanek's Tumor" after his classic description of submucosa granuloma with eosinophilic infiltration ${ }^{7}$.

IFPs are uncommon lesions of the gastrointestinal tract (GIT). The peak age incidence occurs between ages 60 and 70 years ${ }^{5}$. The largest review till date confirmed previous documented reports that the stomach is the most favoured site followed by the small intestine and the colon with reports from the anus and rectum still something of a rarity ${ }^{5}$. However, recent advances in diagnostic techniques especially the widespread use of colonoscopy, has enabled identification of small and asymptomatic polyps, and reports of IFP of the colon have been increasing ${ }^{2}$.

Most patients with IFPs of the GIT are asymptomatic as many are often discovered during routine radiologic or endoscopic tests ${ }^{3}$. Though the symptoms of IFP presence are non-specific at presentation ${ }^{5}$, colonic IFPs can be related to abdominal pain (54\%), bloody stools (33\%), weight loss $(21 \%)$, diarrhea and anemia $(17 \%)^{8}$. The case here presented highlights some clinical traits of a typical patient, as he is 59 years old and was asymptomatic at presentation. The discovery of a rectal lesion is rare and has, to the best of our knowledge, not been presented in any patient from our region.

IFPs are inflammatory tumours growing in the submucosa of the GIT (usually solitary and non- encapsulated). Microscopically, they are characterized by increased proliferation of mononucleate and monomorphic, bland, spindle, and stellate cells all wound within a fibromyxoid stroma with the dominant inflammatory infiltrate being eosinophils? . Regularly also, network of variably sized blood vessels are present ${ }^{10}$.

Both the endoscopic and pathologic findings in this index patient are most consistent with this diagnosis as colonoscopy showed a $3 \mathrm{~mm}$ polypoidal rectal lesion with dilated blood vessels at the surface and the biopsied sample revealed other microscopic features as described earlier (Figure 2).

The definitive diagnosis of large bowel IFP rests on pathological examination ${ }^{2}$. Tissue derived from endoscopic samples had previously been noted to be often unhelpful for the diagnosis of IFPs ${ }^{11}$, but this is changing as aware- ness is increasing and many more of these lesions are incidentally encountered ${ }^{2}$. The case presented illustrates how that endoscopic biopsies along with pathologic examination can be used to clinch diagnosis.

Currently, an IFP is considered to be entirely benign has no malignant potential and metastasis is yet to be report$\mathrm{ed}^{1}$.The small incidental lesions can be excised ${ }^{4}$. However, some complications can develop due to the sheer size of the tumour (e.g. intussusceptions in the small bowel) and these often require surgery- endoscopic or otherwise ${ }^{2,3}$. The patient was counselled about the possibility of recurrence $^{11}$ and has since agreed to come for regular repeat screening endoscopies.

\section{Conclusion}

This case report documents a most uncommon GIT IFPs. It is unique in the fact that it is a first from a region of Africa and we used the case as a signet to review a few pertinent clinical and diagnostic aspects of the disease.

\section{Conflict of interest}

None to declare.

\section{References}

1. Stolte M, Finkenzeller G. Inflammatory fibroid polyp of the stomach. Endoscopy. 1990;22(5):203-7. DOI: 10.1055/s-2007-1012848.

2. Hirasaki S, Matsubara M, Ikeda F, Taniguchi H, Suzuki S. Inflammatory fibroid polyp occurring in the transverse colon diagnosed by endoscopic biopsy. World J Gastroenterol. 2007; 13(27): 3765-3766. DOI: 10.3748/wjg.v13. i27.3765.

3. Nakase H, Mimura J, Kawasaki T, Itani T, Komori H, Hashimoto K, et al. Endoscopic resection of small inflammatory fibroid polyp of the colon. Intern Med. 2000; 39: 25-27 PubMed. DOI: http://doi.org/10.2169/internalmedicine.39.25.

4. Shimura T, Kataoka H, Sasaki M, Kubota E, Shiraki S, Matsusako K, et al. Rectal Inflammatory Fibroid Polyp Resected with EndoscopicSubmucosal Dissection. Inter Med. 2008; 47:2029-2031 PubMed. DOI: http://doi. org/10.2169/internalmedicine.47.1357.

5. Liu TC, Lin MT, Montgomery EA, Singhi AD. Inflammatory fibroid polyps of the gastrointestinal tract: spectrum of clinical, morphologic, and immunohistochemistry features. Am J Surg Pathol. 2013;37(4):586-92. DOI: 10.1097/PAS.0b013e31827ae11e.

6. Helwig EB, Ranier A. Inflammatory fibroid polyps

African Health Sciences Vol 16 Issue 3, September 2016 
of the stomach. Surg Gynecol Obstet. 1953;96:335-367 PubMed .

7. Vanek J. Gastric submucosal granuloma with eosinophilic infiltration. Am J Pathol. 1949;25(3):397-411.

8. de la Plaza R, Picardo AL, Cuberes R, Jara A, MartinezPenalver I, Villanueva MC, et al. Inflammatory fibroid polyps of the large intestine. Dig Dis Sci 1999;44:18101816.

9. Wille P, Borchard F. Fibroid polyps of intestinal tract are inflammatory-re- active proliferations of CD34-posi- tive perivascular cells. Histopathology. 1998;32(6):498-502. PubMed

10. Kolodziejczyk P, Yao T, Tsuneyoshi M. Inflammatory fibroid polyp of the stomach: a special reference to an immunohistochemicalprofile of 42 cases. Am J SurgPathol. 1993;17(11):1159-1168.

11. Zinkiewicz K, Zgodzinski W, Dabrowski A, Szumilo J, Cwik G, Wallner G. Recurrent inflammatory fibroid polyp of cardia: a case report. World J Gastroenterol. 2004;10:767-768. DOI: 10.3748/wjg.v10.i 